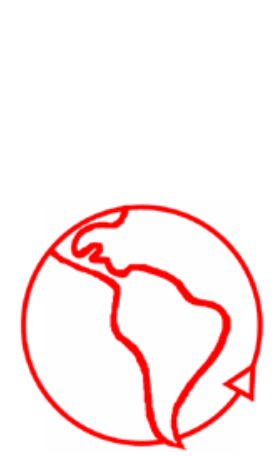

\title{
El desarrollo de un programa de posgrado universitario en un entorno de sistematización: El caso del programa de posgrado en antropología social de la Universidad Iberoamericana, Ciudad de México
}

\author{
The Development of a Graduate Social Anthropology Program in an \\ Systematization Environment: The Social Anthropology Program, at \\ the Iberoamericana University, Mexico City
}

\section{Marisol Pérez Lizaur}

Departamento de Ciencias Sociales y Políticas, Universidad Iberoamericana, México

\section{Resumen}

\begin{abstract}
Con el modelo luhmaniano de organizaciones, en este artículo se analiza el devenir de un programa de posgrado de una universidad jesuita en México. En él se muestra a las universidades como organizaciones modernas en continua interrelación con otros sistemas tanto internos, como los programas de posgrado, como externos, tales como el Estado, los centros financieros y la Compañía de Jesús. Estas relaciones generan conflictos en que los distintos actores, académicos, estudiantes, administrativos y patronos se enfrentan y se acoplan con la finalidad de reproducir la organización
\end{abstract}

Palabras Clave: Universidad; Organización; Sistematización; México; Programa de Posgrado

\begin{abstract}
With the luhmannian model this paper analyses the history of a graduate program in a Mexican Jesuit university. It considers universities as modern organizations interrelated within and outside systems, such as university programs, the State, financial centres and the Company of Jesus. These relationships engender conflict between the different social actors involved in the process: academicians, students, managers and patrons. In such a process actors confront themselves and settle differences so as to reproduce the organization.
\end{abstract}

Keywords: University; Organization; Systematization, Mexico, Graduate Program

\section{Introducción}

Este trabajo fue elaborado inicialmente dentro del proyecto "Antropología de la antropología" (ADELA) dirigido por los doctores Esteban Krotz y Ana Paula de Teresa. Su objetivo inicial fue escribir la historia del programa de posgrado en antropología social de la Universidad Iberoamericana. En este trabajo, asumo que la universidad es una organización (ver Luhmann 2010: 212) y analizo la información histórica desde el punto de vista del análisis de las organizaciones, con base en la teoría de Luhmann (2010).

Según Luhmann las organizaciones son los agentes sociales característicos de la sociedad moderna. Para él una organización es un proceso concatenado de toma de decisiones, orientado a su reproducción, es decir, es un proceso autopoiético. Estos conceptos 
implican que la modernidad busca la sistematización de las actividades sociales y que las organizaciones son los agentes responsables de llevar a cabo dicha sistematización. Según este mismo autor, las organizaciones son parte y motor de la evolución social, a la que se acoplan a través de planes y programas, pero que los cambios diseñados por dichos programas encuentran la oposición del mismo sistema que tiende a reproducirse a sí mismo (Luhmann 1978, 2010).

Las universidades son las organizaciones encargadas de crear, enseñar y difundir los conocimientos de manera sistemática en la sociedad y están en continua interrelación con los sistemas que la integran. Las universidades al mismo tiempo que sistematizan lo que ocurre en los distintos sistemas, en sus procesos internos, toman de sus entornos, externos e internos, las perturbaciones que sintonizan con sus operaciones organizacionales para tener éxito (Arnold 2002). Dicho en otras palabras, las universidades son organizaciones características de la sociedad moderna que al mismo tiempo que inciden en ella, su devenir está condicionado por nichos interaccionales internos, como los grupos de académicos, que pueden perturbar el orden interno, pero que al mismo tiempo pueden convertirse en un capital para sus operaciones transformadoras (cf. Arnold 2002: 18). Los procesos organizacionales de selección de las perturbaciones del entorno que sintonizan con sus operaciones son complejos y generan contradicciones y conflictos (Arnold 2002).

En este trabajo trataré de mostrar la complejidad y dinámica de estos procesos a través de la historia de un programa de posgrado de una universidad privada, de los jesuitas, en México. Es decir, analizaré la historia del Programa de Posgrado en Antropología Social de la Universidad Iberoamericana A.C., destacando los actores que han participado en su formación y desenvolvimiento, sus interrelaciones, los conflictos, así como la manera en que los profesores y estudiantes que lo integran han ido respondiendo a las perturbaciones del entorno, convirtiéndose a su vez en motores de cambio y permanencia en la universidad. En el relato enfatizaré en los conflictos generados por las tendencias sistematizadoras de la sociedad y de la universidad, en particular, frente a la búsqueda de flexibilidad de los académicos, para quienes la sistematización representa un impedimento para la creatividad.

La información para este trabajo fue obtenida a través de entrevistas a los profesores del programa, jesuitas, administradores universitarios y algunos funcionarios encargados de la política de CyT. Así como también, en las bases de datos, los archivos y documentos históricos de la universidad y del programa, su página web, los estatutos y reglamentos de la universidad, así como en bibliografía sobre el programa, la universidad y la educación jesuita. La experiencia de la propia autora como estudiante, profesora de tiempo, coordinadora y senadora universitaria, así como la de Tania Arce como estudiante, becaria y asistente en el proyecto de "Antropología de la antropología" fueron fundamentales para la redacción de este documento

\section{Orígenes (1960-1970)}

La Universidad Iberoamericana fue fundada en 1943 con el nombre original de Centro Cultural Universitario, que forma parte del conjunto de universidades, ubicadas en países de todos los continentes, que han sido confiadas a la Compañía de Jesús ${ }^{1}$ y el programa de licenciatura se abrió en 1960 por iniciativa del Dr. Felipe Pardinas S.J., doctor en Filosofía y egresado de la Escuela Nacional de Antropología e Historia, en 1960, incorporada a la Universidad Nacional Autónoma de México (UNAM).

A finales de la década de 1960 algunos antropólogos mexicanos iniciaron una crítica al proyecto indigenista llevado a cabo por sus congéneres en alianza con los gobiernos

\footnotetext{
${ }^{1}$ http://www.uia.mx/web/site/tpl-Nivel2.php?menu=mgPerfil\&seccion=mgPerfil
} 
revolucionarios. Las críticas a este proyecto surgieron en varios frentes (Krotz 2006: 94; Palerm 1988), uno de ellos fue presidido por el Dr. Ángel Palerm, a quien su experiencia en la Organización de Estados Americanos le hizo reflexionar sobre la importancia de la antropología social para entender los procesos de cambio, su dirección e instrumentación. Para él la antropología social era una ciencia que había acumulado una gran cantidad de información, pero que no había logrado enunciar una teoría a partir de ello, por lo que consideraba necesario proseguir con la investigación empírica y la comparación de sus resultados, para así estar en posición de predecir y orientar los cambios sociales (Palerm 1987:14-19). En colaboración con el Dr. Aguirre Beltrán elaboró un proyecto de programa para formar antropólogos calificados para la investigación y la instrumentación del cambio social (Viqueira 2000).

Este proyecto se fincó en cinco ejes principales: la formación teórica, la investigación empírica, la vinculación de la enseñanza con la investigación, la reflexión y discusión teórica y de los materiales de campo en grupo - los seminarios- y la flexibilidad. Por flexibilidad entendía un programa personalizado, es decir elaborado según los intereses del estudiante. Para Palerm (1988: 351), el currículo de un antropólogo era un concepto informal, no sistematizado, es decir: "Desde muchos puntos de vista el currículo no debería ser más que una declaración general sobre lo que debe saber un estudiante Y de ahí la relativa indiferencia de los antropólogos... Ante las presiones constantes para traducir en un currículo formal lo que para ellos es una serie de ejercicios que combinan la praxis con la teoría y el método, en un marco de realidades objetivas externas a la universidad"

La finalidad del Dr. Felipe Pardinas S.J. al fundar la Escuela de Antropología Social (EAS) en la Universidad Iberoamericana, fue la de "contribuir a orientar la antropología mexicana según una de las tendencias tradicionales más importantes y valiosas, la representada por la inclinación a emplear y aplicar el conocimiento científico social al estudio y solución de los problemas del país" (Palerm 1988: 332). En 1965 los doctores Pardinas, González Rodríguez y Hernández Ornelas, los tres miembros de la Compañía de Jesús, invitaron a Palerm a impartir clases y en 1967 a instrumentar su proyecto de formación de antropólogos, el cual coincidía con sus objetivos. La instrumentación del proyecto implicó la libertad jurídica de la institución, de suerte que pudiera dar títulos, desincorporada de otras instancias ${ }^{2}$; una estructura departamental universitaria ${ }^{3}$, reformas al programa de licenciatura y su articulación con los estudios de grado y la investigación (Palerm 1988). La viabilidad del sistema departamental estuvo apoyada, administrativamente, por un eficiente sistema de cómputo que buscaba la sistematización ${ }^{4}$.

Su instrumentación fue paulatina y fue posible gracias a la similitud de intereses del Dr. Palerm y de los jesuitas, especialmente con el Dr. Ernesto Meneses S.J., un pedagogo, quien fuera rector de 1968 a $1977^{5}$. Coincidieron en su metodología pedagógica, es decir,

\footnotetext{
${ }^{2}$ En 1976 la Secretaría de Educación Pública, mediante el acuerdo 2876 otorgó validez oficial a los estudios superiores de la Universidad Iberoamericana y en 1982 se ratificó la validez de los estudios en todo el territorio mexicano (http://www.uia.com).

3 El sistema departamental se caracteriza porque los departamentos son centros disciplinarios de investigación y docencia, que colaboran entre si para la docencia e investigación. Según la página web la universidad se organizó por departamentos académicos en 1975 (http://www.uia.com), aunque en 1973 el proyecto de departamentalización de la UIA sirvió de base para el diseño del proyecto de la Universidad Autónoma Metropolitana (información personal de MSP).

${ }^{4}$ A pesar de que Palerm valoraba la institucionalización del sistema departamental, por otro lado, criticaba la sistematización, en cuanto implicaba la estandarización de los procesos de enseñanza, lo cual iba en contra de la flexibilidad, para él fundamental para la creación científica.

${ }^{5}$ Durante este periodo la universidad generó una estructura departamental y una de gestión que la independizó de las decisiones académicas de los empresarios, integrantes de su patronato y algunos jesuitas promovieron la fundación de
} 
la enseñanza a partir de la experiencia y de la reflexión ${ }^{6}$, así como también en sus objetivos. Los jesuitas, a nivel mundial, influenciados por la Teología de la Liberación que se desarrollaba en México y otros países de América Latina, optaron como objetivo de su misión, por la búsqueda de la justicia a nivel internacional, no solamente desde la reflexión sino a través de la acción ${ }^{7}$ (Tripole 1999: 7). Para ellos, la raíz de la injusticia era estructural, en lo que coincidían con el modelo teórico marxista que caracterizó el pensamiento teórico de Palerm.

La puesta en marcha del programa se inició con la apertura de la Escuela de Graduados (1966), con un programa libre de maestría y doctorado. El segundo paso fueron ${ }^{8}$ las reformas al programa de licenciatura y su desincorporación de la UNAM (1967). Desde su inicio el programa de posgrado estuvo centrado en la investigación de campo y enfocado a atraer como estudiantes no solamente antropólogos, sino también egresados de otras disciplinas. Debido a la escasez de profesores locales de primer nivel y a la constante penuria económica que caracterizaba a la institución (Palerm 1988: 355), se diseñaron estrategias para atraer profesores destacados, nacionales y extranjeros que pudieran servir como maestros y directores de tesis. El programa se convirtió en el semillero donde se formaron muchos de los maestros e investigadores que posteriormente formaron la base del Centro de Investigaciones y Estudios Superiores en Antropología Social (CIESAS), el Departamento de Antropología de la Universidad Autónoma Metropolitana (UAM-I) y el Colegio de Michoacán, entre otros (García Mora 1988).

\section{Década de 1980 a 1990}

Mientras la década de 1970-1980 fue de crecimiento e innovación, la siguiente fue problemática. Cuatro eventos críticos condicionaron el desempeño del programa: 1) El terremoto del 14 de marzo de 1979 que colapsó las instalaciones de la universidad en Cerro de las Torres; 2) La muerte del Dr. Palerm en junio de 1980; 3) La crisis económica de México; 3) La competencia de otras escuelas de antropología, gratuitas y dependientes del Estado y 4) El hecho de que las Ciencias Sociales dejaron de ser atractivas a los estudiantes provenientes de las clases media alta y alta.

Desde el punto de vista financiero, el terremoto y la crisis económica, aunados a las tendencias de independencia académica de la universidad, con respecto a su patronato, acrecentaron los ya tradicionales problemas económicos de la universidad ${ }^{9}$, de manera que cada día se hizo más difícil la contratación de nuevos profesores y el financiamiento de la investigación. En este panorama, la competencia de otras instituciones que ofrecían la posibilidad de estudios de licenciatura y de grado en forma gratuita, así como el desinterés, entre las clase media alta y alta por las ciencias sociales, incidió en la disminución del número de estudiantes de licenciatura, hasta hacer poco rentable el programa de licenciatura en el marco de la administración universitaria. Como

\footnotetext{
un sindicato que apoyara y defendiera los derechos e intereses de los trabajadores, incluidos los académicos (Palerm 1988; http://www.uia.com).

“"La pedagogía ignaciana se describe en pocas palabras...en la experiencia, reflexión y acción en el aula” (cf. Metts 1997: 29).

${ }^{7}$ Para el padre Arrupe, general de la Compañía en aquel tiempo, la búsqueda de justicia no se queda en la búsqueda de las explicaciones y del desarrollo, sino en la acción. En esta visión tuvieron mucha influencia los jesuitas mexicanos. Unido a este concepto aparece el término de liberación y la participación política. Estos conceptos se derivaron en una orientación de los jesuitas hacia la acción en búsqueda de la justicia más que a la reflexión e investigación académicas. Estos conceptos tendrán consecuencias importantes para la universidad y para el programa de antropología en los años subsecuentes.

${ }^{8}$ El programa se inició como Doctorado en Antropología y en 1976 se cambió por el de Doctorado en Ciencias Sociales con especialidad en Antropología Social.

${ }^{9}$ En 1980 en un terreno en Santa Fe donado por el gobierno federal y con fondos propios, del patronato y de donaciones de particulares se inició la construcción del nuevo campus (http://www.uia.com).
} 
consecuencia de ello, a pesar de su calidad académica, las autoridades universitarias optaron por su cierre en 1980.

Desde el punto de vista académico, la muerte del Dr. Palerm generó un cambio en el liderazgo del programa, aunque se mantuvo vigente el programa de posgrado. A raíz de ello el rector de la universidad solicitó a Dra. Viqueira, esposa de Palerm, doctorada en antropología por la misma universidad (1977) y directora del Departamento de Antropología, que prosiguiera con el de posgrado en antropología social.

Con la finalidad de solucionar los problemas económicos que aquejaban la universidad y al mismo tiempo, estar en posibilidades de construir un nuevo campus, la administración universitaria tomó varias medidas administrativas que repercutieron en el desempeño académico de la institución y del programa. La primera y más significativa fue el cobro por créditos $^{10}$, lo que encareció notablemente los servicios. En el caso del posgrado en antropología ello significó perder estudiantes, ya que muchos de ellos no tenían la capacidad económica de cubrir las colegiaturas. La segunda, fue la unión de los departamentos, en este caso el de Sociología y Antropología en el Departamento de Ciencias Sociales y Políticas. Y en tercero, instrumentaron una política de eliminar plazas de profesores de tiempo.

Mientras esto ocurría en la universidad, en el seno de la Compañía de Jesús en México surgió un conflicto. Por un lado, los seguidores de la teología de la liberación orientados a la acción sociopolítica (cf. Maloney 1999: 273), por el otro, los más proclives a aceptar las recomendaciones de los miembros del patronato a fin de mantener viva la universidad ${ }^{11}$. Como en toda organización, los conflictos de sus dueños o líderes afectaron su operación (Gersick et. al. 1997). En este marco los funcionarios universitarios mostraron una preferencia por la ciencia política y la sociología más cercanas a la acción política, que a la reflexión teórica y a la investigación empírica, con resultados a largo plazo, característicos del programa de posgrado en antropología social. El programa no era autofinanciable, representaba una carga a las finanzas universitarias y su flexibilidad lo hacía administrativamente difícil de controlar y estaba integrado por maestros y estudiantes muy críticos. Las entrevistas a los profesores que vivieron esa época dan cuenta de que estos conflictos se expresaron en un golpeteo constante. Las posiciones de dirección quedaron en manos de los sociólogos y de los politólogos con grados académicos menores que los de los profesores de antropología, a quienes trataron de eliminar.

A pesar de este marco institucional poco propicio, con el liderazgo de la Dra. Viqueira y el apoyo de los profesores, integrados en un Colegio, el programa logró un reforzamiento de los estudios teóricos, conservar la matrícula, proseguir con la invitación a maestros distinguidos y ampliar la planta académica en gran medida gracias al apoyo del sindicato y de la rectoría. Cada maestro tenía su proyecto de investigación en los se integraban los alumnos, como si fueran especialidades. La orientación teórica de la investigación fue esencialmente la misma, aunque se dio una gran variedad de temas de investigación. A pesar de los conflictos, en este lapso se graduaron 19 maestros y 5 doctores y se publicaron varios libros con temas novedosos.

El costo de los estudios era alto, lo cual dejaba fuera a muchos estudiantes y el problema se incrementó con la mudanza al nuevo campus de Santa Fe en 1988. Ante dicha situación, en 1987 los maestros del programa optaron por recurrir a las becas del Consejo Nacional de Ciencia y Tecnología (CONACYT). Esta institución otorgaba becas individuales, de colegiatura y manutención, a estudiantes que se postulaban ante los comités. Fueron

\footnotetext{
${ }^{10}$ Unidad de valor o puntuación de una asignatura (UIA 2005: 34)

${ }^{11}$ Este conflicto y la fuerza de los objetivos en búsqueda de la justicia y acercamiento con los pobres de la Compañía de Jesús se expresó en el cierre del Instituto Patria, en 1970-71, escuela en donde se formaba a los hijos de la élite de la ciudad de México, con la finalidad de librar recursos para obras prioritarias.
} 
las primeras becas que esta institución otorgó a estudiantes de la UIA, la que además abrió un fondo de becas para los estudiantes de posgrado. Gracias a esos fondos se logró ampliar y diversificar la matrícula.

Uno de los puntos que mayores enfrentamientos generaba el programa con la administración universitaria era su flexibilidad, difícil de controlar con los sistemas de cómputo y opuesta a la sistematización. El programa se organizaba con base a la investigación y a los seminarios, es decir espacios en los "que se transmite la cultura, es decir lo que se ha escrito, leyendo a estos autores que han trabajado antes" (Viqueira, información verbal). Los costos se calculaban por créditos; los estudiantes tenían que pagar por los seminarios, la atención de los profesores y el uso de las instalaciones y servicios universitarios, pero no así por los créditos de investigación de campo, en cuanto éstos no significaban costo a la universidad. Sin embargo, a raíz de las crisis económicas de 1980 los estudiantes tuvieron que pagar por todo, lo que dejó fuera a la mayoría de los posibles estudiantes, ya que provenían en su mayoría, de estratos medios.

Ante dicha situación el Colegio de Profesores, en el que tenían presencia fuerte los estudiantes, reconoció la necesidad de adaptarse a los requisitos de sistematización y control que demandaban el sistema de cómputo universitario y el CONACYT, con el fin de asegurar el financiamiento para los estudiantes, por lo que acordó un cambio en el programa. Este cambio consistió en señalar un número de créditos, que incluían los de investigación, que había que cumplir para obtener los grados. A diferencia de otros programas de posgrado, se prosiguió con la política de abrir semestralmente las inscripciones, ya que la experiencia de los maestros mostró que las interrelaciones de los estudiantes de distintos grados y experiencias, enriquecía la vida académica.

\section{La década de la institucionalización: 1990- 2000}

Esta década, a nivel mundial, se caracterizó por tendencias evolutivas orientadas hacia el capitalismo financiero. A raíz de las crisis económicas que afectaron especialmente a América Latina, durante la década de 1980, los grandes organismos supranacionales como el Fondo Monetario Internacional y el Banco Mundial instrumentaron políticas de corte neoliberal a fin de remediarlas. Estos "remedios" implicaron un incremento en los procesos de globalización, especialmente la financiera, la puesta en marcha de los tratados de libre comercio, así como la implantación de sistemas que permitieran el control y la transparencia en el manejo de los fondos públicos. La instrumentación de estas políticas fue posible, gracias a la revolución tecnológica que hizo posible la expansión de los sistemas de cómputo y el uso común del Internet. Los gobiernos mexicanos se unieron a dicha corriente y embarcaron al país en esas políticas que implicaban políticas de eficiencia y transparencia en el uso de los recursos. Estas medidas afectaron las políticas de Ciencia y Tecnología nacionales y en consecuencia las de la Universidad Iberoamericana. En ese contexto el CONACYT optó, entre otras medidas, por una política de promoción de los posgrados con base en la evaluación de los programas de acuerdo a criterios de calidad ${ }^{12}$, muy ligados con los de eficiencia y atar las becas a dichos programas, en vez de otorgarlas en forma personal. Así surgió el Padrón Nacional de Posgrados ${ }^{13}$.

Ante la escasez de recursos universitarios y alerta de las dificultades económicas de los estudiantes, el Colegio de Profesores optó por inscribir el programa en el Padrón de Excelencia del CONACYT en 1993, en el que fue aceptado. Gracias a la inscripción en el

\footnotetext{
${ }^{12}$ Inicialmente los criterios de calidad fueron de un orden mas cualitativo y con los años y el desarrollo de la tecnología se tornaron cuantitativos. Otro criterio, muy ligado con los tratados internacionales fue la posibilidad de los intercambios académicos, con bases de homogeneidad en los programas.

${ }^{13}$ Esta política ha sido altamente criticada, ya que no está fundamentada en un proyecto nacional de posgrados (Arredondo).
} 
padrón, el número de estudiantes creció, ya que tuvieron acceso a becas, que en ese entonces cubrían la colegiatura y la manutención. Con la finalidad de cumplir con los requisitos del CONACYT el programa apoyó a los maestros de tiempo para finalizar sus doctorados y prosiguió invitando a maestros distinguidos.

Durante los primeros cinco años de la década se acentuaron los problemas financieros de la universidad, lo que se expresaba en un mayor conflicto en el departamento de Ciencias Sociales. No obstante, el programa, la organización de la investigación y la relación entre estudiantes y profesores se mantuvo, de tal manera que su trabajo se expresó en la graduación de 28 maestros y 13 doctores y varias publicaciones. La variedad de las temáticas desarrolladas en las tesis refleja la filosofía del programa sobre la formación personalizada de investigadores, así como también el interés por nuevas temáticas. Sin embargo, las tesis muestran una diferencia con los intereses que empezaron a dominar la antropología mexicana del momento: la sociedad multicultural, los grupos indígenas, la política y la subjetividad (De la Peña 2002; Krotz 2006).

Mientras que el programa de posgrado en antropología sorteaba, gracias al esfuerzo de sus profesores los problemas de la universidad, la institución se enfrentaba a ellos, especialmente los financieros con una gran dificultad. Los jesuitas, el patronato, algunos investigadores y maestros realizaron estudios sobre la situación de la universidad que Ilamaron la atención sobre los problemas vigentes, especialmente la disminución de la matrícula, la baja en la calidad académica, la escasa sistematización del posgrado y la poca eficiencia del personal. En busca de una solución en 1996 nombraron rector al maestro en educación Enrique González Torres S.J., jesuita, quien se había distinguido por sus relaciones con grupos empresariales y su capacidad de organización. Este nuevo rector asumió el rectorado con la misión de rescatar financieramente la universidad y de elevar el nivel académico.

Por otro lado, en la Compañía de Jesús también se daban procesos de cambio, relacionados con la hegemonía de las universidades estadounidenses en el conjunto de instituciones de educación jesuitas. ${ }^{14}$ El peso político de estas instituciones en las políticas globales de la Compañía se expresó entre otras cosas, en que el director general de educación superior de los jesuitas era estadounidense, así como en las tendencias de buscar el uso eficiente de los recursos invertidos en la educación superior con base en la sistematización y la transparencia. Según el Presidente de una universidad jesuita estadounidense, las instituciones jesuitas de educación superior requieren emplear las herramientas de la administración moderna con el fin de hacer más eficientes los recursos, disminuir costos y elevar la calidad, ya que son básicos para lograr las metas apostólicas de la Compañía, que incluyen la investigación académica y la difusión del conocimiento (Piderit S.J.1999: 46). El nuevo rector conocía bien estos conceptos y tenía claro que para alcanzar las metas que le habían señalado tenía que apoyarse en el empleo de la administración moderna, que implica sistematización y eficiencia, así como en sus redes sociales.

Varias fueron las medidas adoptadas para alcanzar los objetivos planteados, aquí nos centraremos en lo político, lo académico y lo administrativo. Desde el punto de vista político, el rector propuso cambios en los estatutos y reglamentos universitarios, con el objetivo de institucionalizar la universidad. El estatuto orgánico (2003) otorgó más poder a los miembros del patronato $\left(\mathrm{FICSAC}^{15}\right.$ ) y abolió los Colegios de Profesores. El reglamento de personal académico enfatizó la importancia de la evaluación de los maestros e investigadores, sin perder el criterio imperante en las universidades jesuitas de no tratar a

\footnotetext{
${ }^{14}$ Hoy día el $18.92 \%$ de las universidades jesuitas están en los Estados Unidos, mientras que el global de las europeas representan el $26.89 \%$ y las latinoamericanas el 30\%. (http://www.sjweb.info/highedu/index.cfm)

${ }^{15}$ FICSAC, integrado por representantes del mundo empresarial, jesuitas y presidentes de instituciones de educación superior jesuitas en los Estados Unidos de América.
} 
los maestros y estudiantes como medios para alcanzar ciertos fines (Piderit 1999:46) ${ }^{16}$. El patronato enfatizó el uso eficiente de los recursos y su rentabilidad, lo que desembocó en el planteamiento de una planeación estratégica, inicialmente realizada en forma participativa y cualitativa, pero posteriormente con la asesoría de un gran despacho de origen estadounidense ${ }^{17}$, con criterios fundamentalmente cuantitativos. Esta política desembocó en una orientación muy pronunciada a la sistematización de todos los procesos universitarios entre ellos el posgrado, la estandarización de los programas y generó una crítica constante a la flexibilidad y falta de sistematización del programa de antropología.

Las políticas administrativas fueron acompañadas por las académicas. Una de sus primeras medidas fue el incorporar sistemas de control de calidad a la universidad, como las evaluaciones de académicos y administrativos, a pesar de que contravenía el espíritu de las instituciones jesuitas (cf. Pederit 1999: 46). Se invitó a los programas de posgrado a ser evaluados por instancias externas como el CONACYT y a los investigadores a incorporarse al Sistema Nacional de Investigadores, a pesar del costo que ello representaba para la institución, ya que el Estado no pagaba las becas de los investigadores que laboran en instituciones privadas ${ }^{18}$. La incorporación del programa de antropología al padrón de excelencia del CONACYT, así como la de sus profesores al SNI convirtió al programa de antropología en el líder de los posgrados de la universidad.

En este marco, el nuevo rector buscó restablecer el orden académico en el departamento de Ciencias Sociales, uno de los más afectados por la crisis universitaria. Para ello nombró un nuevo director, en 1996, al Dr. Javier Torres Nafarrate, quien reorientó la actividad del departamento hacia la investigación académica y propició la comunicación entre los académicos. En este nuevo ambiente los profesores del programa de posgrado en antropología encontraron un líder académico que valoró de inmediato la calidad y perspectivas académicas del mismo. A pesar de que el nuevo estatuto orgánico eliminó los Colegios de Profesores, el director apoyó su existencia, así como las decisiones emanadas del mismo, las cuales son ratificadas por el Consejo técnico, el órgano oficialmente reconocido.

En 1997, a sugerencia de un profesor del programa y con el apoyo del Colegio de Profesores y del rector, el director nombró como coordinadora del programa de posgrado en antropología social a una ex-alumna del mismo, con experiencia de trabajo y de investigación en políticas de $\mathrm{CyT}^{19}$. El rector se ocupó personalmente de encomendarle la tarea proseguir con el programa de posgrado, aumentar la matrícula, ampliar y rejuvenecer la planta académica, proseguir con la investigación, pero incorporando nuevas temáticas, así como conseguir su ratificación en el Padrón de Programas de Excelencia del CONACYT, vital para que los estudiantes pudieran obtener becas y continuar con el programa.

En 1997 el programa fue ratificado, pero condicionado, en el Padrón de Excelencia del CONACYT. El condicionamiento fue con base al compromiso de ampliar la planta académica y de tener más profesores en el SNI. Con base en ello, se aseguraron las becas

\footnotetext{
${ }^{16}$ Los nuevos estatutos y reglamentos fueron fuertemente criticados por la comunidad universitaria. Por un lado, contravenían el espíritu del Ideario escrito por el rector Meneses y por otro, porque no se adecuaban totalmente a las modernas políticas de evaluación imperantes en otras instituciones. Las políticas de este nuevo rector fueron también altamente criticadas en cuanto que para instrumentarlas recurrió a sistemas considerados como autoritarios.

${ }_{17}$ Uno de los miembros del patronato era un alto ejecutivo de McKinsey, compañía consultora en sistemas para empresas y promotora de la homologación de sistemas alrededor del mundo.

${ }^{18}$ Actualmente el Estado paga el $33 \%$ de las mismas y las instituciones el $66 \%$ restante.

${ }^{19}$ El Dr. Palerm mencionaba que la mejor opción para la gestión de un programa académico eran sus propios egresados después que hubieran pasado al menos veinte años trabajando en otras instancias académicas, a fin de reproducirlo, pero enriqueciéndolo con nuevas experiencias.
} 
de los estudiantes, pero sin embargo, la política del CONACYT había cambiado y las becas ya no incluían la manutención, lo que implicó que los estudiantes no fueran de tiempo completo ya que tenían que trabajar para vivir. Esta circunstancia representó un nuevo reto ya que la mayoría de los estudiantes eran maestros de instituciones de educación superior públicas de provincia. A pesar de ello la matrícula creció. El reconocimiento, aunque condicionado, del programa de posgrado ratificó la posición de liderazgo del programa en la universidad y representó una ventaja en la negociación de apoyos y recursos para el programa frente a la universidad ${ }^{20}$.

Dentro del proyecto de reestructurar el departamento de Ciencias Sociales y Políticas y de renovación y ampliación de la planta académica el rector nombró, por sugerencia de los profesores de antropología, a la Dra. Carmen Bueno Castellanos, ex alumna del programa, con experiencia en otras instituciones, como la primera directora antropóloga del departamento. Con ella el número de maestros de tiempo completo ascendió a seis, pero independientemente del número, el programa se enriqueció notablemente con los intereses de investigación de los nuevos profesores y se reforzó la tradición teórica y metodológica del programa alrededor del materialismo histórico y el énfasis en el trabajo de campo. Los nuevos aires imperantes se reflejaron en una mayor demanda y matrícula escolar aunque persistió el conflicto con la universidad alrededor de la flexibilidad y la sistematización.

\section{Los nuevos aires y dilemas del nuevo siglo (2000-2005)}

La experiencia de la Dra. Bueno en otras instituciones se reflejó en la instrumentación de sistemas que facilitaron la institucionalización del departamento y de sus programas en un ambiente de intercambio académico dinámico y ordenado. Los órganos colegiados oficiales como el comité académico y los consejos técnicos, así como los "no oficiales" como los colegios de profesores, funcionaron en una forma más constante y eficiente y se incrementó el número de profesores jóvenes, con doctorados en instituciones de prestigio, a pesar de su costo. La presencia de nuevos maestros con formación e intereses comunes a la antropología acercó una vez más al departamento de ciencias sociales a su concepción inicial, es decir un espacio en donde investigadores de diversas disciplinas sociales trabajaran conjuntamente en la investigación y formación de nuevos investigadores. Su presencia fue la base para reforzar y enriquecer la vida académica y contar con una planta con los requisitos cuantitativos que el CONACYT exige: Ocho para maestría y doce para doctorado.

La vitalidad académica del programa de antropología se expresó en publicaciones, la graduación de 10 doctores y 14 maestros en dos años y en la incorporación de cinco de los investigadores al SNI. Sin embargo, a pesar de ello los conflictos entre la administración universitaria y el programa de antropología, generados por su falta de sistematización y excesiva flexibilidad, llegaron a su clímax. Ante esta situación el Colegio de Profesores y el Consejo Técnico optaron por un cambio en el programa, procurando organizarlo alrededor de los cinco ejes que caracterizaron el programa de Palerm: la formación teórica, el trabajo de campo, la vinculación de la enseñanza con la investigación, la reflexión y discusión teórica y de los materiales de campo en grupo - los seminarios- y la flexibilidad ${ }^{21}$; pero que al mismo tiempo presentara la estructura requerida por la Secretaría de Educación Pública, la Dirección de Servicios Escolares de la universidad, así como que tuviera un menor costo para los estudiantes. El programa fue elaborado por todos los maestros y fue aprobado por la SEP en 2004.

\footnotetext{
${ }^{20}$ El General de la Compañía mencionó: "queremos graduados que estén dispuestos a ser líderes preocupados por la sociedad y el mundo, deseosos de acabar con el hambre y los enfrentamientos; que capten la necesidad de una más equitativa distribución de la magnificencia de Dios...... En resumen, queremos que nuestros graduados sean líderes para servir" (P. Peter Hans Kolvenbach, S.J.).

${ }^{21}$ Esta flexibilidad implica entre otras cosas la posibilidad de abrir las inscripciones semestralmente.
} 
El programa de doctorado fue calificado por el CONACYT en 2005 como de nivel internacional y el de maestría igualmente en 2011. Gracias a dichos reconocimientos los estudiantes tienen derecho a becas del CONACYT, así como de otras instancias. La vitalidad y calidad académica del programa son actualmente reconocidas no solamente por instancias externas a la universidad, sino también por la propia institución ${ }^{22}$. Tan es así que los programas son reconocidos como líderes dentro de la institución y sus profesores consultados por los directivos de los otros programas para lograr los reconocimientos del CONACYT. Ello no implica que la universidad apoye ampliamente al programa y que no se den conflictos con las autoridades actuales, especialmente en lo que se refiere a la adjudicación de tiempos para la investigación y la dirección de tesis. Y en el Colegio de Profesores subsiste la duda sobre si la adaptación del programa a los nuevos lineamientos ${ }^{23}$ asegura la calidad de los trabajos de graduación.

Y esto ocurre en un entorno globalizante que entre otras cosas ha puesto de "moda" la certificación internacional de las universidades y la búsqueda de la homologación de sus programas. En este entorno el nuevo rector, el Dr. José Morales Orozco S.J. ha escuchado positivamente las sugerencias del patronato universitario, muy afín con las tendencias globales de educación superior, para promover la certificación internacional y la homologación de los programas. Ello ha implicado la consulta con una certificadora estadounidense de educación superior, así como en un énfasis en la planeación estratégica, orientada a adecuarse a las metas de la certificadora. Sin embargo, el proceso ha sido difícil, ya que las condiciones de la universidad no corresponden con las de las universidades estadounidenses, especialmente en lo que se refiere al porcentaje de profesores de tiempo y sus responsabilidades. Este proceso ha generado conflictos en la institución, pero especialmente, entre el programa de posgrado en antropología y las autoridades universitarias. Uno de los temas que ha generado los mayores roces entre ambas es el hecho de que la certificadora exige que los profesores de tiempo impartan clases en los programas de licenciatura y esta tarea implica la distracción de los profesores del programa de sus tareas básicas: la dirección de tesis de posgrado y la investigación, labores indispensables para conservar la certificación del CONACYT y del SIN, básicas para la sobrevivencia del programa.

La nueva directora, La Dra. Helena Varela, politóloga, ha invitado a los profesores del programa a dar clases en la licenciatura, a cambio de un apoyo total al posgrado. Sin embargo, el conflicto permanece y el Colegio de Profesores sigue peleando por el tiempo y dedicación de sus miembros a sus tareas específicas.

\section{Conclusiones}

En este apartado mostraré desde el punto de vista de la teoría de las organizaciones de Luhmann algunas conclusiones que permiten explicar el desarrollo del Programa de Posgrado en Antropología Social y su sobrevivencia en un entorno a veces hostil.

El análisis de la historia del programa de posgrado en antropología social de la Universidad Iberoamericana, con base en la propuesta de Luhmann, permite apreciar en primer lugar, que las universidades son organizaciones que son parte de la sociedad y que son agentes importantes en los procesos de sistematización característicos de la globalización y por ende de la sociedad actual.

\footnotetext{
${ }^{22}$ La coordinadora del programa fue invitada a participar en el comité universitario de posgrado, y los directores de departamento y coordinadores de otros posgrados consultan a la directora y a los coordinadores del programa de antropología sobre la manera de entrar y permanecer en el PNP.

${ }^{23}$ La sistematización implícita en el nuevo programa implica ajustarse a los tiempos que da el CONACYT para medir la eficiencia terminal: tres años para maestría y cuatro y medio para el doctorado.
} 
En segundo, que las universidades son organizaciones que a través de las decisiones de sus integrantes buscan su reproducción o sobrevivencia en un entorno en continua evolución. También muestra que las universidades son parte de sistemas mayores, en este caso el de la educación superior en México y del sistema de educación superior de la Compañía de Jesús. En ese sentido, a veces las decisiones se orientan a la permanencia en uno de esos sistemas, mientras que en otras, tiende a su propia reproducción. Proceso durante el cual, las organizaciones, en este caso la Universidad Iberoamericana, toman de las perturbaciones del entorno externo o interno aprendizajes que le son necesarios para su propia sobrevivencia. Así encontramos que en el momento de dificultades económicas, la universidad toma de la Compañía de Jesús medidas administrativas que en otra situación no habría hecho, porque perturbaban su propio devenir.

Al mismo tiempo, esta historia da cuenta que la Universidad Iberoamericana como organización está en continua interacción con otros sistemas externos a ella, que le generan perturbaciones. Estos son, entre otros, el económico, el político y aún el natural, en cuanto que un terremoto fue capaz de destrozar sus instalaciones, frente a los cuales tiene que tomar decisiones para su asegurar su reproducción.

En este sentido el Programa de Posgrado en Antropología Social aparece como un sistema interno de la propia organización, es decir, como parte de su entorno interno. La posibilidad de observarlo como tal, permite apreciar la manera en que cada sistema, en este caso la universidad y el programa, son sistemas que buscan su propia reproducción, a pesar de que paradójicamente pertenezcan a una misma organización. Tal como ocurre con la Universidad Iberoamericana frente a los sistemas a los que pertenece.

La posibilidad de analizar el Programa de Posgrado en Antropología Social como un sistema en relación con otras organizaciones y sistemas, es decir, la Universidad Iberoamericana, la Compañía de Jesús, el CONACYT, la Antropología Mexicana, el sistema económico y aun el natural, permite apreciar que efectivamente es una organización en cuanto toma decisiones encaminadas a su propia reproducción. $Y$ aparece evidente que toma de sus entornos aquello que permite su propia reproducción. Un ejemplo de ello, es el momento cuando sus profesores deciden cambiar sus programas, aun a costa de flexibilidad, para asegurar su sobrevivencia y reproducción. Mientras que por su parte, la universidad aprende del programa, a pesar de las perturbaciones y los conflictos que le genera. De esta manera, reconoce su experiencia de acoplarse a otros sistemas, al consultar a sus miembros acerca de la forma de permanecer en el PNP. Esta interacción ayuda a ambos sistemas en su reproducción.

Otro aprendizaje importante derivado de este tipo de análisis se refiere al cambio organizacional. Esta pequeña historia da cuenta de que los programas y proyectos de cambio estructural no son siempre aquellos que favorecen la sobrevivencia de las organizaciones. Un ejemplo de ello es el cambio en los estatutos universitarios que hacen desaparecer los Colegios de Profesores. En este caso, los profesores, con el apoyo de sus directores, conservan su participación e interacción en el Colegio. Y es precisamente esta decisión la que permite la toma de decisiones colegiada de los profesores, cuyas decisiones, a su vez, permiten el desarrollo del programa y logran incidir en las decisiones de la universidad. Es más, es precisamente esta posibilidad de no cambio, la gran herramienta del programa para enfrentarse y permanecer, aun ante las amenazas de las certificadoras externas y del patronato.

Luhmann (2010: 415-436) arguye que el desarrollo de la técnica, especialmente de la computación y la informática son parte de la evolución social, y que como tal, inciden en las organizaciones. Sin embargo, también discute el hecho de que no es la técnica la que genera los cambios organizacionales, sino que son las decisiones de sus integrantes, quienes ven en la técnica una herramienta para la supervivencia de la organización. La historia aquí analizada es un ejemplo de esta situación. La información da cuenta de que 
es la administración universitaria la que decide utilizar la computación para facilitar la sistematización y el acoplamiento de la organización con otros sistemas. Por su parte, el programa tomó de esta perturbación del entorno, técnicas y métodos que le han permitido acoplarse a los sistemas externos como el CONACY y la propia universidad, pero al mismo tiempo conservando su flexibilidad.

Finalmente, considero que la posibilidad de hacer un análisis organizacional de un programa de posgrado como el que aquí elaboro, con el enfoque luhmaniano, permite apreciar, en primer lugar, que las universidades no son organizaciones, como se pretende desde la mirada de algunos administradores, no conflictivas, sino por el contrario, altamente complejas y conflictivas. $Y$ en segundo, que los cambios planeados no son siempre el mejor camino para la sobrevivencia y desarrollo de las organizaciones, antes bien, las tendencias de permanencia, muchas veces son las que lo aseguran.RM

\section{Bibliografía}

Arnold, M. (2002). Las organizaciones sociales como sistemas autopoiéticos. Mimeo.

Arredondo, V. (s.f.). La situación actual del posgrado en México. Manuscrito.

Compañía de Jesús. URL: http://www.sjweb.info/highedu/index.cfm

De la Peña, G. \& Vázquez, L. (comps.) (2002). La antropología sociocultural en el México del milenio: Búsquedas, encuentros y transiciones. México D.F.: FCE.

Gersick, K. A., Davis, J. A., Hampton, M. M., \& Lansberg, I. (1997). Generation to Generation: Life Cycles of the Family Business. Boston, Mass: Harvard Business School Press.

Krotz, E. (2006). Mexican anthropology's Ongoing Search for Identity. En L. Ribeiro \& A. Escobar (Eds.), World Anthropologies (pp. 87-109). Oxford/ New York: Berg.

Luhmann, N. (2010). Organización y decisión. México D.F.: Universidad Iberoamericana.

Maloney, T. (1999). The GC43 and the Jesuit Political Scientist. En M. Tripole (Ed.), Promise Renewed. Jesuit Higher Education for a New Milenium (pp. 273- 295). Chicago: Loyola Press.

Metts, R. (1997). Ignacio lo sabía. La pedagogía jesuita y las corrientes educativas actuales. Guadalajara: ITESO

Palerm, A. (1987). Introducción a la Teoría Etnológica. Querétaro: Universidad Autónoma de Querétaro.

Palerm, A. (1988). Escuela de antropología Social de la Universidad Iberoamericana. En C. García Mora (coord.), La antropología en México. Panorama histórico. Vol. 7 Las instituciones (pp. 332-356). México D.F.: Colección Biblioteca del INAH. Instituto Nacional de Antropología e Historia.

Pérez Lizaur, M. (2007). Presentación de los libros de Historia de la Etnología de Ángel Palerm: Los precursores, Los evolucionistas, Tylor y los profesionales británicos. Iberofórum, 2(3).

Piderit, J. (1999). Managing Jesuit Universities Alter GC 34. En M. Tripole (Ed.), Promise Renewed. Jesuit Higher Education for a New Milenium (pp. 43-61). Chicago: Loyola Press.

Sánchez Saldaña, M. (2004). La universidad privada frente a los retos del posgrado. Revista OMNIA, (núm. esp.), 201- 207.

Tripole, M. (1999). An Assesment of the 34th General Congregation's Understanding of Justice and Its role in Jesuit Higher Education. En M. Tripole (Ed.), Promise Renewed. Jesuit Higher Education for a New Milenium (pp. 1- 26). Chicago: Loyola Press.

Universidad Iberoamericana (1977). Comunicación oficial no. 108.

Universidad Iberoamericana (2003). Estatuto orgánico. México: Mimeo.

Universidad Iberoamericana (2003). Reglamento de posgrado. Mimeo.

Universidad Iberoamericana (2005). Reglamento de estudios de licenciatura.

Universidad Iberoamericana (2006). Reglamento de investigación. 
Universidad Iberoamericana (2012). Historia. (URL: http://www.uia.mx/web/site/tplNivel2.php?menu=mgPerfil\&seccion=anHistoria Jun/2013)

Viqueira Landa, C. (2000). Algunas aportaciones de Ángel Palerm a la enseñanza de la Antropología. En V. García Acosta (coord.), La diversidad intelectual. Ángel Palerm in memoriam (pp. 115-126). México D.F.: CIESAS.

\section{Sobre la autora}

Marisol Pérez Lizaur es profesora del Departamento de Ciencias Sociales y Políticas de la Universidad Iberoamericana, México. Doctora en Antropología Social por la Universidad Iberoamericana de la Ciudad de México. Entre sus áreas de especialización se encuentran: estudios sobre universidades, élites, organizaciones, empresas y empresarios. Entre sus publicaciones se destacan: Empresa y familia. Una visión desde la antropología (compiladora, Universidad Iberoamericana, 2010), Una familia de la élite mexicana (con Larissa Adler Lomnitz, Alianza Editorial, 2006) e Historia de las universidades estatales (con J. Castrejón, SEP, 1975).

\section{Contacto}

Universidad Iberoamericana

Departamento de Ciencias Sociales y Políticas

Prolongación Paseo de la Reforma 880

Lomas de Santa Fe

0210 México D.F.

marisol.perez@uia.mx

Recibido: Marzo 2013 Aceptado: Junio 2013 